\title{
Effect of High Energy Ball Milling on the Structure and Phase Decomposition of the Multiferroic $\mathrm{Bi}_{5} \mathrm{Ti}_{3} \mathrm{FeO}_{15}$ Ceramics
}

\author{
J. Dercz ${ }^{a, *}$, M. Zubko ${ }^{b}$, G. Dercz ${ }^{b}$, I. MatuŁA ${ }^{b}$ \\ ${ }^{a}$ Institute of Technology and Mechatronics, University of Silesia, Żytnia 12, 41-200 Sosnowiec, Poland \\ ${ }^{b}$ Institute of Materials Science, University of Silesia, 75 Pułku Piechoty 1A, 41-500 Chorzów, Poland \\ The paper presents the results of the $\mathrm{Bi}_{5} \mathrm{Ti}_{3} \mathrm{FeO}_{15}$ multiferroic phase stability analysis during high-energy \\ ball milling aimed at obtaining fine dispersion ceramic powder. The X-ray diffraction and transmission electron \\ microscopy methods were used to analyse the structure and verify the degree of crystallite dispersion. Structural \\ data analysis was carried out using the Rietveld method. To carry out the analysis of the morphology, the scanning \\ electron microscopy was used. The results that were obtained showed that the high energy ball milling process \\ results in the decomposition of the initial ceramics, where finally $\mathrm{Bi}_{5} \mathrm{Ti}_{3} \mathrm{FeO}_{15}$ and $\mathrm{Bi}$ are obtained. An increase \\ in the proportion of the amorphous phase and an increase in the dispersion of the grains and crystallites of the \\ powder that occurs with an increase in the milling time were observed.
}

DOI: 10.12693/APhysPolA.130.852

PACS/topics: 75.85.+t, 61.05.cp, 68.37.Lp, 81.20.Wk

\section{Introduction}

The family of smart materials is very large. Most often they are produced in the form of single crystals, polycrystals, (e.g. ceramic materials) and composites as well as in the form of thin films or multilayered "sandwich"-type systems $[1,2]$. Among the smart materials, ferroics, biferroics, and multiferroics are of high importance. They are characterized by unique physical properties [3-10]. In 1994 Schmid as multiferroics called the materials which simultaneously displayed at least two ferroic states [11]. Materials in which there was a simultaneous arrangement of magnetic and electric moments were called ferroelectromagnetics. The most perspective ferroelectromagnetics include bismuth oxides with the perovskitelike layered structure (BLPO). Due to the lack of oxygen, in bismuth-based perovskites there occurs the deformation of the crystallite structure, which consists in the formation of the so-called layered structures. One of the examples of layered structures are the Aurivillius phases $[12,13]$. Bismuth oxides with the perovskitelike layered structures are composed of regularly structured $\left(\mathrm{Bi}_{2} \mathrm{O}_{2}\right)^{2+}$ bismuth layers intertwined with the $\left(\mathrm{A}_{m-1} \mathrm{~B}_{m} \mathrm{O}_{3 m+1}\right)^{2-}$ perovskite packages. Spaces A are occupied by big cations (such as $\mathrm{Ba}, \mathrm{Sr}, \mathrm{Ca}, \mathrm{Bi}$, and $\mathrm{Pb}$ ), spaces $\mathrm{B}$ are occupied by small cations of transition metals ( $\mathrm{Ti}, \mathrm{Nb}, \mathrm{Ta}, \mathrm{Mo}, \mathrm{W}, \mathrm{Fe}$, and $\mathrm{Mn})$; whereas $m$ denotes the number of oxygen octahedrons arranged layer by layer, along the axis of the $(m=1,2,3$, $4,5,8)$ perovskite package. An example multiferroic with a layered bismuth perovskite-like structure is the $\mathrm{Bi}_{5} \mathrm{Ti}_{3} \mathrm{FeO}_{15}$ (BTF) ceramics. The first description of the crystal BTF structure was performed in 1992 by Kubel

\footnotetext{
*corresponding author; e-mail: jolanta.dercz@us.edu.pl
}

and Schmid [14]. They attributed to it an orthorhombic system with the Fmm2 space group and the lattice parameters of $a_{0}=5.4318(6) \mathrm{nm}, b_{0}=41.149(4) \mathrm{nm}$, and $c_{0}=5.4691(12) \mathrm{nm}$ (the main symmetrical axis is axis $c$ ).

Application possibilities of multiferroics primarily depend on the degree of mutual coupling of individual subsystems, both the magnetic and electric ones. Therefore, ceramics with high density and high purity must be obtained, which is directly related to the properties of ceramics.

High energy ball milling (HEBM) is an effective way of obtaining nanocrystallite and amorphic materials with unique properties due to the fact that during the process of milling ceramic powders are deformed and fractured many times. During the HEBM process, the surface activity of the milled powders is enhanced due to the crash and shock, and - after milling - the surface activity of powders is in the metastable high-energy state. Fine dispersion powders obtained in this way may be used in the synthesis of ceramics with high density as well as to obtain thin film multilayered systems $[3,4,7]$.

The main purpose of this paper is to determine the stability of the multiferroic BTF phase during high-energy ball milling, aiming at preparation of fine dispersion ceramic powder. Moreover, structural changes of the powder as well as the size of the crystallites and the lattice distortion at each milling stage were also determined.

\section{Experimental details}

As a starting material for milling, multiferroic single phase ceramics with the composition of BTF (ICDD PDF 82-0063) obtained by a solid state reaction process from mechanically activated precursors was used [7]. The pellets obtained in this way, within the framework of this study, were subjected to high-energy ball milling in the Fritch PULVERISETTE 7 premium line ball mill. Containers and balls (with the diameter of $5 \mathrm{~mm}$ ) made of 
hardened steel were used, and the weight ratio of balls to the powder was 10:1. Ball milling was performed at a rotational speed of $500 \mathrm{rpm}$ and was identical for all samples. In order to minimize the oxidation, the process was carried out in the argon atmosphere. The isopropyl alcohol of $3 \mathrm{wt} \%$ was used as the process control agent (PCA) to keep the balance between cold welding and fracturing of powders during the milling process.

The most important variable of mechanical synthesis is the milling time. It is responsible for the final dispersion and phase composition of powders. For research purposes, the powders were milled for the following times: 50,100 , and $150 \mathrm{~h}$.

The crystalline structure and phase content of the obtained milled and sintered materials were tested by $\mathrm{X}$ ray diffraction with the use of an X-ray X'Pert diffractometer manufactured by Phillips, with copper target Xray tube $(\lambda=1.54 \AA)$ operated at $40 \mathrm{kV}$ and $30 \mathrm{~mA}$. $\mathrm{Cu} K_{\alpha}$ radiation was monochromized with a graphite monochromizer. WAXS curves were recorded in a step scanning mode, over a difraction angle $(2 \theta)$ range: $10^{\circ}$ $140^{\circ}$, with the step of $0.05^{\circ}$.

Refinement of the Bragg-Brentano X-ray diffraction pattern was carried out using Rietveld's whole X-ray profile fitting technique with the DBWS 9807a program. The profile function used to fit the theoretical diffraction curves to the experimental ones was the pseudoVoigt $[15,16]$. The weight fraction of each component was determined from the optimized scale factors using the relation proposed by Hill and Howard [17]. The size of the crystallites and the lattice distortion were measured by the Willamson-Hall method. Verification tests of the structure and size of crystallites were conducted using the electron transmission microscope JEM 3010 operating at the accelerating voltage of $300 \mathrm{kV}$.

\section{Results}

Qualitative phase analysis of the material milled for 50 and $100 \mathrm{~h}$ showed that we deal with a one-phase powder (Fig. 1).

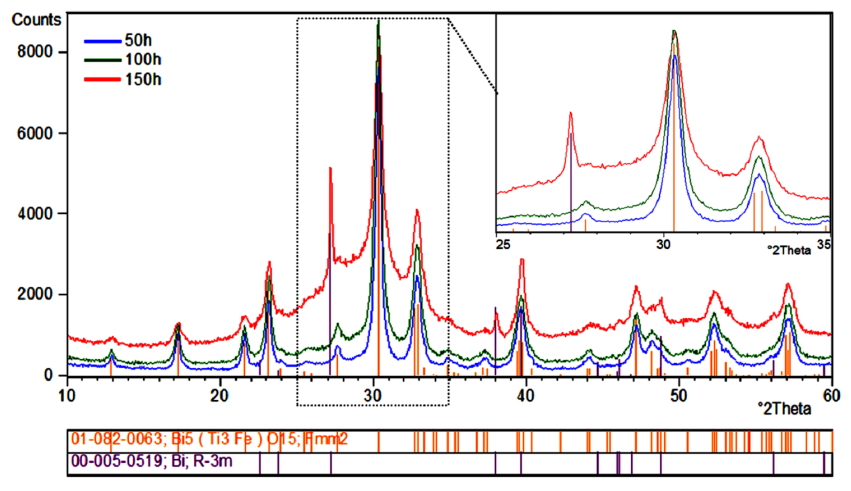

Fig. 1. The diffraction patterns of BTF ceramics milled for 50, 100, and $150 \mathrm{~h}$.

Phase BTF (ICDD PDF 01-082-0063) with the Fmm2 space group was identified, which corresponds to the initial state of ceramics used to milling. For the material with the longest milling time $(150 \mathrm{~h})$, decomposition of ceramics was identified and the formation of the $\mathrm{Bi}$ (ICDD PDF 00-005-0519) phase, which underwent the process of crystallisation in the $R \overline{3} m$ space group, was observed.

Detailed analysis of the resulting diffraction patterns revealed a progressive process of amorphisation of the powder during the subsequent milling stages. This is particularly evident for the longest milling time (Fig. 1). In addition, there was an increase in the width of the diffraction lines, which proved an increase in both the level of the nanocrystallisation of the crystallites as well as the lattice distortions (Table I).

TABLE I

Changes of average crystallite size $(D)$ and lattice distortion $(\langle\Delta a / a\rangle)$ of phases of the milled samples.

\begin{tabular}{c|c|c|c|c}
\hline \hline & & \multicolumn{3}{|c}{ Milling time $[\mathrm{h}]$} \\
\cline { 3 - 5 } Phase & Parameters & 50 & 100 & 150 \\
\hline \multirow{2}{*}{$\mathrm{BTF}$} & $D[\mathrm{~nm}]$ & $51(4)$ & $44(3)$ & $14(2)$ \\
& $\langle\Delta a / a\rangle$ & $6.36 \times 10^{-3}$ & $7.65 \times 10^{-3}$ & $7.99 \times 10^{-3}$ \\
\hline \multirow{2}{*}{$\mathrm{Bi}$} & $D[\mathrm{~nm}]$ & - & - & $65(6)$ \\
& $\langle\Delta a / a\rangle$ & - & - & $5.24 \times 10^{-3}$
\end{tabular}

It was found that milling for the two shortest times (50 and $100 \mathrm{~h}$ ) causes the nanocrystallisation of the BTF ceramics, reaching an average size of the order of $50 \mathrm{~nm}$. At the same time, a slight increase in the lattice distortion was observed. For the longest time, the crystallites size is reduced to $14(2) \mathrm{nm}$ (Table I). Lattice distortion is then in the range typical for the materials after milling process. In the case of bismuth, the estimated size of the crystallites was in the range of $65(6) \mathrm{nm}$.

The Rietveld analysis helped to determine the lattice parameters of the phases occurring at different milling stages and in the initial BTF ceramics. In the case of the BTF phase it was found that the highest degree of deformation of the unit cell occurs after $150 \mathrm{~h}$ of milling (Table II). This might be due to the decomposition of ceramics by separation of the $\mathrm{Bi}$ phase as a result of the nanocrystallisation with simultaneous amorphisation of ceramics. In the case of $\mathrm{Bi}$ in the sample after $150 \mathrm{~h}$ of milling, a decrease in the size of the unit cell was also observed. There were no additional diffraction lines from the phases of the remaining elements of the initial BTF ceramics.

The effect of the long-term milling on the powder morphology after 50 and $150 \mathrm{~h}$ of milling was studied using a transmission electron microscopy (TEM). The analysis of the obtained images in a bright and dark field and of the corresponding selected area electron diffraction (SAED) pattern proved the nanocrystalline nature of the obtained material after milling. Observation in the bright and dark field of powder after the shortest milling time clearly indicates that powders consists of grains formed from single crystalline multiferroic ceramics. The distribution of grain size range from 50 to $100 \mathrm{~nm}$. 
TABLE II

Lattice parameters of the phases of the milled sample.

\begin{tabular}{c|c|c|c|c|c|c}
\hline \hline \multirow{3}{*}{ Phase } & Lattice & & \multicolumn{4}{|c}{ Milling time $[\mathrm{h}]$} \\
\cline { 4 - 7 } & param. & ICDD & 0 & 50 & 100 & 150 \\
\hline \multirow{3}{*}{$\mathrm{BTF}$} & $a_{0}[\mathrm{~nm}]$ & 0.5432 & $0.5437(6)$ & $0.5435(7)$ & $0.5438(8)$ & $0.5397(8)$ \\
& $b_{0}[\mathrm{~nm}]$ & 4.1149 & $4.1349(4)$ & $4.1151(6)$ & $4.1150(6)$ & $4.1113(6)$ \\
& $c_{0}[\mathrm{~nm}]$ & 0.5490 & $0.5447(6)$ & $0.5466(9)$ & $0.5463(9)$ & $0.5461(8)$ \\
\hline \multirow{2}{*}{$\mathrm{Bi}$} & $a_{0}[\mathrm{~nm}]$ & 0.4548 & - & - & - & $0.4530(4)$ \\
& $c_{0}[\mathrm{~nm}]$ & 1.1852 & - & - & - & $1.1820(5)$ \\
\hline
\end{tabular}

* International Centre for Diffraction Data ${ }^{\circledR}$

The high resolution TEM (HRTEM) picture (Fig. 2b) obtained for powder grain after $50 \mathrm{~h}$ of milling shows structure characteristic for oxygen layers of the BTF ceramics. The SAED pattern (Fig. 2c) confirms single crystalline character with interplanar distance characteristic for the BTF ceramics.

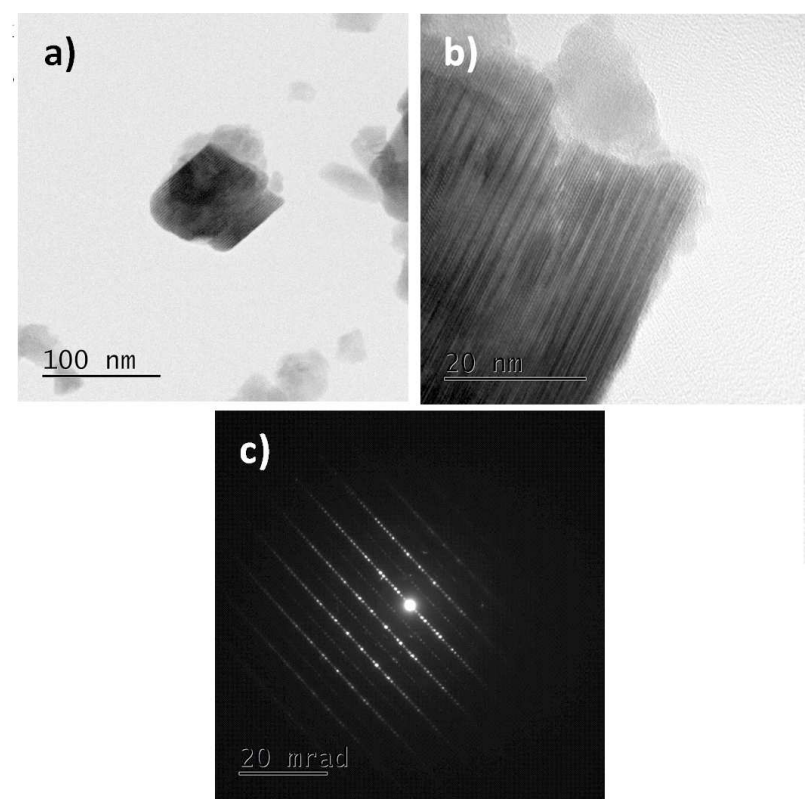

Fig. 2. The TEM image of BTF ceramics milled for $50 \mathrm{~h}$ : (a) and (b) are the TEM bright and dark field images, respectively, (c) the corresponding SAED pattern.

The bright and dark field images (Fig. 3a,b) of powder after $150 \mathrm{~h}$ of milling time clearly indicate that observed grains consist of agglomerated nanocrystals. Grains size measurements performed on the dark field images showed that size distribution is in the $10-20 \mathrm{~nm}$ range. Moreover, corresponding SAED patterns (Fig. 3c) revealed also presence of amorphous fraction.

After milling, SEM images (Fig. 4) of the ceramics showed increased dispersion of the powder particles when the milling time was increased. At the same time, the degree of agglomeration of the particles was reduced. The size of the particles was from $10 \mu \mathrm{m}$ to $1 \mu \mathrm{m}$ for 50 and $150 \mathrm{~h}$ of milling, respectively.

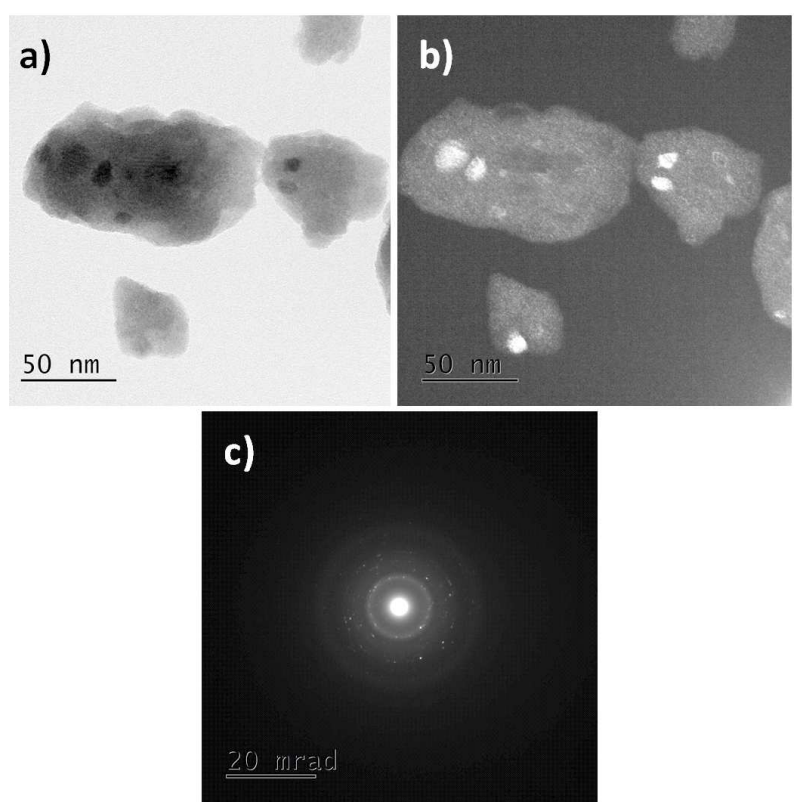

Fig. 3. The TEM image of BTF ceramics milled for $150 \mathrm{~h}$ : (a) and (b) are the TEM bright and dark field images, respectively, (c) the corresponding SAED pattern.

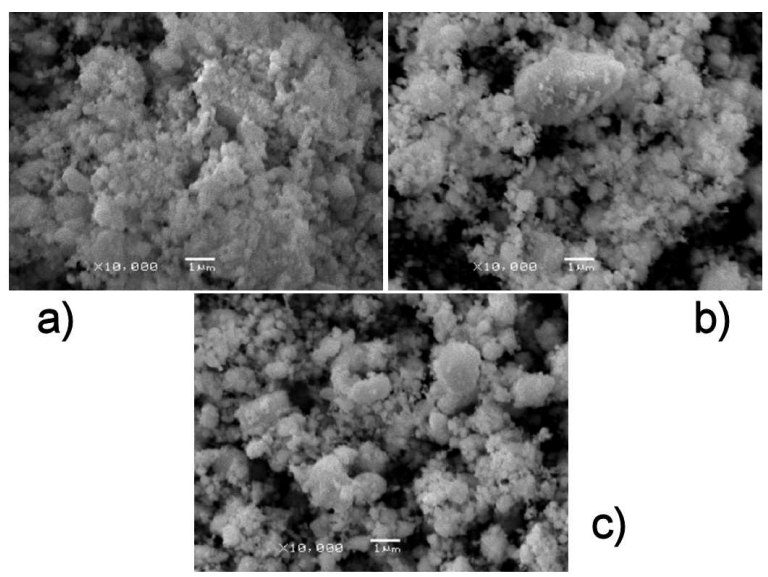

Fig. 4. The SEM images of BTF ceramics milled for: (a) 50, (b) 100, and (c) $150 \mathrm{~h}$.

\section{Conclusion}

1. The effect of the high-energy milling time on the structure and phase decomposition of the BTF multiferroic ceramics was observed.

2. X-ray and electron microscopy studies are a proof of the presence of the BTF multiferroic ceramics as nanocrystalline phases. Moreover, the amorphisation of ceramics is observed.

3. It was discovered that the optimal long-term highenergy milling time is $100 \mathrm{~h}$. Fine nanocrystalline single phase multiferroic BTF ceramics was obtained. 


\section{Acknowledgments}

This work was partially supported (milling process) by the Polish National Science Centre (in Polish: Narodowe Centrum Nauki, NCN) under the research project no. UMO-2011/03/D/ST8/04884.

\section{References}

[1] A. Lawver, Smart Mater. Bull. 8, 5 (2001).

[2] A.P. Douvalis, M. Venkatesan, P. Velasco, C.B. Fitzgerald, J.M.D. Coey, J. Appl. Phys. 93, 8071 (2003).

[3] J. Dercz, J. Bartkowska, G. Dercz, P. Stoch, M. Łukasik, Int. J. Thermophys. 34, 567 (2013).

[4] J. Bartkowska, J. Dercz, J. Exp. Theor. Phys. 117, 875 (2013).

[5] A. Hill, A. Filippeti, J. Magn. Magn. Mater. 242, 976 (2002).

[6] Y. X. Liu, J.G. Wan, J.M. Lin, C.W. Nan, J. Appl. Phys. 94, 5118 (2003).
[7] J. Dercz, A. Starczewska, G. Dercz, Int. J. Thermophys. 32, 746 (2011).

[8] M.M. Kumar, V.R. Palkar, K. Srinivas, S.V. Suryanarayana, Appl. Phys. Lett. 76, 2764 (2006).

[9] X.W. Dong, K.F. Wang, J.G. Wan, J.S. Zhu, J.M. Liu, J. Appl. Phys. 103, 94 (2008).

[10] M. Kumar, K.L. Yadav, G.D. Varma, Mater. Lett. 62, 1159 (2008).

[11] H. Schmid, Ferroelectrics 162, 317 (1994).

[12] B. Aurivillius, Arkh. Khemi. 1, 499 (1949).

[13] B. Aurivillius, Arkh. Khemi. 2, 512 (1950).

[14] F. Kubel, H. Schmid, Ferroelectrics 129, 101 (1992).

[15] H.M. Rietveld, J. Appl. Crystallogr. 3, 65 (1969).

[16] R.A.Young, The Rietveld Method, Oxford Univ. Press, New York 1993.

[17] R.J. Hill, C.J. Howard, J. Appl. Crystallogr. 20, 467 (1987). 